
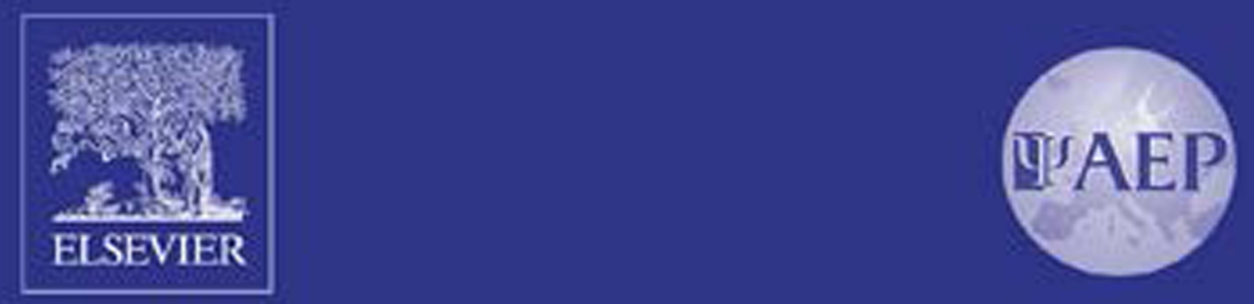

ISSN 0924.9338

January 2007

$$
\text { Vol. } 22 \text { - No. } 1 \text { - p. } 1-68
$$

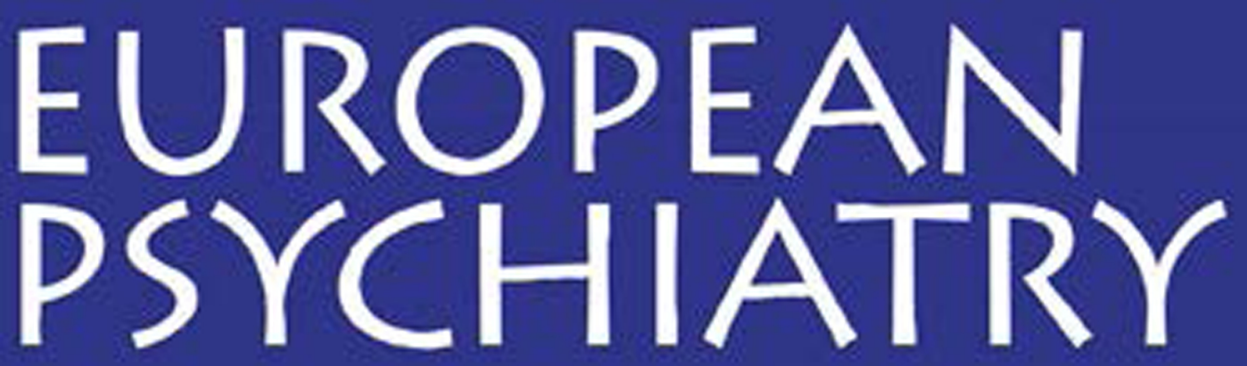

THE IOURNAL OF THE ASSOCIATION OF EUROPEAN PSYCHIATRISTS

1 Relative efficacy of psychotherapy and combined therapy in the treatment of

depression: A meta-analysis

S.M de Mart, $J$, Doldir, RA Schoovers and fi de vonghe

9 Problem solving therapies for depression: A meta-analysis P. Qujeers, A van Straten and L Warmerdem

\title{
GENETIC EPIDEMIOLOGY
}

AND ITS METHODS

16 Famillal aggregation of depression, but no famillal aggregation of individual dopressive symptoms $R$ Hevo and S. Heen

22. Paternal ages bolow or above 35 years old are associated with a different risk of schizophrenia in the ottspring $M$ Wosiand $P$ Conwod

27 Temperament and character dimensions of the relatives of schizophrenia patients and controls: The relotionship between schizotypal features and personality E Bora and 8. Vernedarose

32 Shared executive dysfunctions in unatlected relatives of patients with autism and obsessive-compulsive disorder

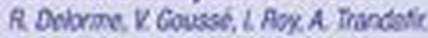
F. Muoniou, M. C. Alouron Simition. C. Betancar and $M$ Lebojer

39 Distribution of 1293A > C polymorphism of methylenototrahydrofolate reductase gene in pabients with bipolar disorder and schizophrenia

Q. Kemoisto. A Bobor. M. Luacoli

R Grersk, A. Stczesankiewcz, d. Howser and PP logodissed

44 Diagnostic Interview for Genetic Studies (OVGS): inter-rater and test-retest rellability and validity in a Spanish population

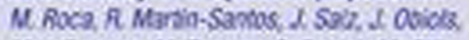

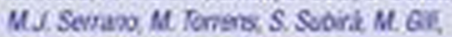
R. Nevines: A Batie, U Nady, $N$ Bumantes and 5 Carleles

49 Validity, roliabinity and objectivity of the family history method in psychiatry: A meta analysis $f$ hurut and P franke LEGAL ISSUES

59 Psychiatric commitment: Over 50 years of case law from the European Court of Human Rights Q. Nwewe asd J. Moteo 


\section{EDITORS}

S. Frangou (London) \& Ph. Gorwood (Paris) \& R. Heun (Birmingham).

Editorial Office: Prof. Ph. Gorwood, Editor of European Psychiatry - INSERM U675, Faculté Xavier Bichat, 16, rue Henri Huchard, 75018 Paris (France). e-mail: european.psychiatry@bichat.inserm.fr - Tel. 33147606415 - Fax. 33147606740.

\section{EDITORIAL BOARD}

P. Boyer (Ottawa), J.D. Guelfi (Paris), M. Maj (Naples), R. Murray (London), H. Sass (Aachen)

\section{STATISTICAL ADVISORS}

A. Heyting (Da Marken), N. Takei (Hamamatsu), B. Falissard (Paris)

\section{ADVISORY BOARD (permanent reviewers)}

Aalto-Setälä, Terhi, Espoo, Finland; Amaddeo, Francesco, Verona, Italy; Arango, Celso, Madrid, Spain; Barbui, Corrado, Verona, Italy; Bellivier, Franck, Créteil, France; Bertschy, Gilles, Geneva, Switzerland; Brambilla, Paolo, Milan, Italy; Broome, Matthew, London, UK; Clark, Luke, Cambridge, UK; Clarke, Mary, Stillorgan, Ireland; Colom, Francesc, Barcelona, Spain; Courtet, Philippe, Montpellier, France; Craddock, Nick, Birmingham, UK; Crocq, Marc-Antoine, Rouffach, France; Dazzan, Paola, London, UK; Delamillieure, Pascal, Caen, France; Deuschle, Michael, Mannheim, Germany; Dollfus, Sonia, Caen, France; Dubertret, Caroline, Colombes, France; Ekselius, Lisa, Uppsala, Sweden; Fabrozzo, Michele, Naples, Italy; Falkai, Peter, Hamburg, Germany; Favaro, Angela, Padova, Italy; Galderisi, Silvana, Naples, Italy; Garcia-Portilla, Maria-Paz, Oviedo, Spain; Giannakopoulos, Panteleimon, Geneva, Switzerland; Gonzales-Pinto, Ana, Vitoria, Spain; Gourion, David, Paris, France; Grabe Hans-Jörgen, Stralsund, Germany; Gustavsson, Petter, Stockholm, Sweden; Hansenne, Michel, Liege, Belgique; Heinz, Andreas, Berlin, Germany; Hosak, Ladislav, Hradec Kralove, Czech Republic; Hrdlicka, Michal, Prague, Czech Republic; Kircher, Tilo, Aachen, Germany; Kirov, George, Cardiff, UK; Kontaxakis, Vassili, Athens, Greece; Kurzthaler, Ilsemarie, Innsbruck, Austria; Lederbogen, Florian, Mannheim, Germany; Licht, Rasmus-Wentzer, Risskov, Denmark; Lieb Roselind, Munich, Germany; MacCabe, James, London, UK; MacGregor-Lawrie, Stephen, Edimburgh, Scotland; Mallet, Luc, Paris, France; Martinot, Jean-Luc, Orsay, France; McDonald, Colm, London, UK; McGuire, Philip, London, UK; Menchon, Jose-Manuel, Hospitalet de Llobregat, Spain; Mico, Juan-Antonio, Cadiz, Spain; Mohr, Pavel, Prague, Czech Republic; Monteleone, Palmiero, Naples, Italy; Mucci, Armida, Naples, Italy; Müller, Ulrich, Cambridge, UK; Murray, Graham, Cambridge, UK; Neeleman, Jan, Groningen, the Netherlands; Nordström, Anna-Lena, Stockholm, Sweden; O'Callaghan, Eadbhard, Dublin, Ireland; Öngur, Dost, Boston, USA; Oswald, Pierre, Brussels, Belgium; Paillere-Martinot, Marie-Laure, Paris, France; Papadimitriou, George, Athens, Greece; Pariante, Carmine M., London, UK; Pélissolo, Antoine, Paris, France; Percudani, Mauro, Milan, Italy; Perez, Jorge, Brescia, Italy; Pilowski, Lyn, London, UK; Pini, Stefano, Pisa, Italy; Pinto, Emmanuel, Liege, Belgium; Pitchot, William, Bertrix, Belgium; Runeson, Bo, Stockholm, Sweden; Sahakian, Barbara, Cambridge, UK; Schmidt, Ulrike, London, UK; Schuman, Gunther, Mannheim, Germany; Schütz, Christian, Bonn, Germany; Schwarz, Marcus, Munich, Germany; Sommer, Wolfgang, Stockholm, Sweden; Souery, Daniel, Brussels, Belgium; Spence, Sean A., Sheffield, UK; Ströhle, Andreas, Berlin, Germany; Suvisaari, Jaana, Helsinki, Finland; Thome, Johannes, Swansea, UK; Tortorella, Alfonso, Naples, Italy; Van Os, Jim, Maastricht, The Netherlands; Verdoux, Hélène, Bordeaux, France; Weiss, Elisabeth M., New York, USA; Yazgan, Yanki, Istanbul, Turkey.

\section{EDITORS EMERITUS}

C. Ballus (Barcelona), P. Bech (Copenhagen), H. Heimann (Tübingen), Y Lecrubier (Paris), C.B. Pull (Luxembourg)

\section{THE JOURNAL OF THE ASSOCIATION OF EUROPEAN PSYCHIATRISTS www.aep.lu}

\author{
President: H. Sass (Aachen) \\ Past President: M. Maj (Naples) \\ President Elect: C. Höschl (Prague) \\ Secretary General: M. Kastrup (Copenhagen) \\ Treasurer: K. Mann (Mannheim) \\ Counsellors: P. Boyer (Ottawa), S. Tyano (Petach-Tikva) \\ Sections: M. Musalek (Vienna)
}

European Psychiatry (ISSN 0924-9338) 2007 (volume 22) One year, 8 issues. France (TTC): 567 Euros. See complete rates on the subscription form included in this issue.

Address order and payment to Elsevier Masson SAS, Service Abonnements, 62, rue Camillie-Desmoulins, 92442 Issy-les-Moulineaux cedex: payment by check or credit card (CB, MasterCard, EuroCard or Visa: indicate number and expiration date); by transfer:

CCP Paris n $30041000011904540 \mathrm{H}$ 020/70.

Subscriptions begin 4 weeks after receipt of payment and start with the first issue of the calendar year. Back issues and volumes are available from the publisher. Claims for missing issues should be made within 6 months of publication. Includes air delivery.

Adversiting - Nicolas Zarjevski. Tel.: (33) 01711651 38. Fax: (33) 01711651 11. E-mail: publ@elsevier.fr

Subscriptions - Tel.: (33) 01711655 55. Fax: (33) 01711655 88. E-mail: abt2@elsevier.fr

Publisher - Annette Doré

General manager and publishing director - Daniel Rodriguez

\section{Author enquiries}

For enquiries relating to the submission of articles (including electronic submission where available) please visit Elsevier's Author Gateway at http://authors.elsevier.com. The Author Gateway also provides the facility to track accepted articles and set up e-mail alerts to inform you of when an article's status has changed, as well as detailed artwork guidelines, copyright information, frequently asked questions and more. Contact details for questions arising after acceptance of an article, especially those relating to proofs, are provided after registration of an article for publication.

Subscription conditions, instructions to authors, the contents of each issue as well as the abstracts of the articles published in European Psychiatry are available on the website of Elsevier France: www.elsevier.fr 